\title{
SUR LES MESURES CONIQUES LOCALISABLES
}

\author{
RICHARD BECKER
}

(Received 13 August 1981)

Communicated by R. Vyborny

\begin{abstract}
Let $X$ be a weakly complete proper cone, contained in an Hausdorff locally convex space $E$, with continuous dual $E^{\prime}$. A positive linear form on the Risz space of functions on $X$ generated by $E^{\prime}$ is called a conical measure on $X$. Let $M^{+}(X)$ be the set of all conical measures on $X$. G. Choquet asked the question: when is every conical measure on $X$ given by a Radon measure on $(X \backslash 0)$ ? Let $\ell$ be the class of such $X$. In this paper we show that the fact that $X \in \ell$ only depends, in some sense, on the cofinal subsets of the space $\left.E^{\prime}\right|_{X}$ ordered by the order of functions on $X$. We derive that $X \in E$ is equivalent to $M^{+}(M) \in \mathcal{L}$. We show that $\mathcal{L}$ is closed under denumerable products.
\end{abstract}

1980 Mathematics subject classification (Amer. Math. Soc.): 46 A 55.

Soit $E$ un espace faible séparé, de dual $E^{\prime}$, et $X$ un cône convexe saillant et faiblement complet contenu dans $E$ (class $\Im$ ). G. Choquet a posé la question de savoir quand toute mesure conique $\mu \geqslant 0$ ([2], Sections 30,38,40) sur $X$ était localisable en une mesure de Radon généralisée sur $(X \backslash 0)$ (c'est à dire: existe t-il une famille de compacts $K_{i} \subset(X \backslash 0)$ et une famille de mesures de Radon $m_{i} \geqslant 0$, où $m_{i}$ est portée par $K_{i}$, telle que $\mu=\sum m_{i}$ ).

On notera $\varrho$ la classe des cônes de $\delta$ qui possèdent cette propriété.

Nous allons montrer ici, qu'en un certain sens, le fait que $X \in \mathcal{E}$ ne dépend que des parties cofinales du polaire $E_{+}^{\prime}$ de $X$.

Au paragraphe 6, on en déduira une conséquence intéressante: si $M^{+}(X)$ désigne le cône des mesures coniques $\geqslant 0$ sur $X$, on a:

$$
(X \in \mathfrak{Q}) \Leftrightarrow\left(M^{+}(X) \in \mathfrak{E}\right) \text {. }
$$

Un cas particulier bien connu est celui où l'origine admet, dans $X$, une base dénombrable de voisinages (classe $\mathfrak{S}_{D}$ ); alors on a aussi $M^{+}(X) \in \mathfrak{S}_{D}$; les cônes

Copyright Australian Mathematical Society 1982 
de $\mathfrak{S}_{D}$ sont bien coiffés au sens de G. Choquet ([2] 30,16,17, 18) et les mesures coniques $\geqslant 0$ qu'ils portent sont localisables en des mesures de Radon $\geqslant 0$, sur des compacts de ces cônes.

Nous allons d'abord nous placer dans le cadre des cônes faiblement complets.

1. Lemme. Soient $X$ et $Y$ deux éléments de $S$, contenus dans des e.l.c.s. E et $F$, et $\varphi$ une application linéaire continue de $E$ dans $F$, telle que $\varphi(X) \subset Y$.

$S i$, pour tout compact $K \subset Y$, on a $\varphi^{-1}(K) \cap X$ compact, alors, pour toute $\lambda \in M^{+}(X)$, les deux propriétés suivantes sont équivalentes:

1) $\lambda$ est localisable en une mesure de Radon généralisée sur $X \backslash 0$.

2) $\varphi(\lambda)$ est localisable en une mesure de Radon généralisée sur $Y \backslash 0$.

Preuve. 1) $\Rightarrow 2$ ). Est presque évident (voir E. Thomas [5]).

$2) \Rightarrow 1$ ). Comme $Y \in S$, tout compact de $Y$ est contenu dans un convexe compact de $Y$. Il en résulte que toute mesure conique $\geqslant 0$ sur $Y$, localisable en une mesure de Radon généralisée sur $Y \backslash 0$ est sup. de mesures coniques localisables sur des convexes compacts de $Y \backslash 0$.

On suppose d'abord que $\varphi(\lambda)$ est localisable sur un convexe compact $K$ de $Y \backslash 0$ : on sait qu'on peut supposer $K \subset u^{-1}(1)$, où $u \in F^{\prime}$. Soit $K^{\prime}=\varphi^{-1}(K) \cap X$; par hypothèse, c'est un convexe compact de $X \backslash 0$, contenu dans $v^{-1}(1)$ où $v=u \circ \varphi$ est un élément de $E^{\prime}$. Alors $\lambda$ est limite de mesures coniques discrètes ([2] 30.9) $\sum_{1}^{n} \varepsilon_{r\left(\lambda_{i}\right)}$ avec des $\lambda_{i} \in M^{+}(X)$ vérifiant $\Sigma_{1}^{n} \lambda_{i}=\lambda$ et $\lambda_{i} \neq 0$.

Notons que $\left(\lambda_{i} \neq 0\right) \Rightarrow\left(\varphi\left(\lambda_{i}\right) \neq 0\right)$, puisque $\varphi^{-1}(0) \cap X=(0)$. Or on a $\sum_{1}^{n} \varepsilon_{r\left(\lambda_{i}\right)}=\sum_{i}^{n} \lambda_{i}(v) . \varepsilon_{r\left(\lambda_{i}\right)} / \lambda_{i}(v)$; le dernier $\Sigma$ peut s'inter-prêter comme une mesure de Radon $\geqslant 0$ sur $K^{\prime}$ puisque $\lambda_{i}(v)=\varphi\left(\lambda_{i}\right)(u)>0, \Sigma_{1}^{n} \lambda_{i}(v)=\lambda(u)$ et que $\varepsilon_{r\left(\lambda_{i}\right)} / \lambda_{i}(v) \in K^{\prime}$. D'où une localisation de $\lambda$ sur $K^{\prime}$, en passant à la limite.

Pour passer au cas général, il reste à montrer que toute $\lambda \in M^{+}(X)$, vérifiant 2ème), peut s'écrire $\lambda=\lambda_{1}+\lambda_{2}$ avec $\lambda_{1} \neq 0$ et $\varphi\left(\lambda_{1}\right)$ localisable sur un convexe compact de $Y \backslash 0$. Pour cela, il suffit d'utiliser le lemme suivant:

2. Lemme. Soient $X$ et $Y$ deux éléments de $\delta$, contenus dans des e.l.c.s. E et $F$, et $\varphi$ une application linéaire continue de $E$ dans $F$, telle que $\varphi(X) \subset Y$. Soient $\lambda \in M^{+}(X)$ et $\mu=\varphi(\lambda)$. Pour toute décomposition de $\mu$ en $\mu_{1}+\mu_{2}$, il existe une décomposition de $\lambda$ en $\lambda=\lambda_{1}+\lambda_{2}$ telle que $\mu_{1}=\varphi\left(\lambda_{1}\right)$ et $\mu_{2}=\varphi\left(\lambda_{2}\right)$.

Preuve. Désignons par $h(X)$ et $h(Y)$ les treillis de fonctions sur $X$ et $Y$ engendrés par $\left.E^{\prime}\right|_{X}$ et $\left.E^{\prime}\right|_{Y}$. On se place dans $h(X) \times h(X)$; soit $p$ la forme sous-linéaire définie sur cet espace, à valeurs dans $R \cup(+\infty)$, telle que:

$$
\begin{aligned}
p\left(f_{1}, f_{2}\right)= & \inf \left(\mu_{1}\left(g_{1}\right) ; g_{1} \in h(Y), g_{1} \circ \varphi \geqslant f_{1}\right) \\
& +\inf \left(\mu_{2}\left(g_{2}\right): g_{2} \in h(Y), g_{2} \circ \varphi \geqslant f_{2}\right) .
\end{aligned}
$$


On voit que $\left(f_{1}, f_{2} \leqslant 0\right) \Rightarrow(p \leqslant 0)$. Soit $\tilde{\lambda}$ définie sur la diagonale de $h(X) \times$ $h(X)$ par: $\tilde{\lambda}(f, f)=\lambda(f)$; on voit que $\tilde{\lambda}$ est majorée par $p$.

D'après la version du théorème de Hahn-Banach due à Dinges [3] (voir aussi [1]), $\tilde{\lambda}$ se prolonge en une forme linéaire $\lambda$ sur $h(X) \times h(X)$, majorée par $p$, telle que $\hat{\lambda}\left(f_{1}, f_{2}\right)=\lambda_{1}\left(f_{1}\right)+\lambda_{2}\left(f_{2}\right)$. Il est clair que $\lambda_{1}, \lambda_{2} \geqslant 0$, puisque, pour toute $f \in h(X)$ on a $p(f, 0) \leqslant 0$ et $p(0, f) \leqslant 0$. Montrons que $\varphi\left(\lambda_{1}\right)=\mu_{1}$ et $\varphi\left(\lambda_{2}\right)=$ $\mu_{2}$ :

Soit $f \in h(Y)$; on a $\varphi\left(\lambda_{1}\right)(f)=\lambda_{1}(f \circ \varphi) \leqslant p(f \circ \varphi, 0) \leqslant \mu_{1}(f)$; d'où $\mu_{1}(f)$ $=\lambda_{1}(f \circ \varphi)$ soit $\varphi\left(\lambda_{1}\right)=\mu_{1}$.

Nous allons indiquer un cas particulier intéressant dans lequel les conditions du Lemme 1 sont vérifiées.

3. Proposition. Soient $X$ et $Y$ deux éléments de $\mathcal{S}$, contenus dans des e.l.c.s. $E$ et $F$, et $\varphi$ une application linéaire continue de $E$ dans $F$, telle que $\varphi(X) \subset Y$. On suppose que dans $E^{\prime}$ ordonné par $X^{\circ}, \varphi^{t}\left(F^{\prime}\right)$ est cofinal à $X^{\circ}$. Alors $\varphi$ est propre.

Preuve. Il faut prouver que $\varphi$ est fermée et que l'image réciproque par $\varphi$ de tout compact est compact.

L'assertion concernant les compacts résulte du fait que, pour toute partie $A$ d'un cône de $\mathcal{S}$, on a ( $A$ compact $) \Leftrightarrow(A$ fermé et borné).

Montrons que $\varphi$ est fermée: soient $A$ un fermé de $X$ et $b \in \overline{\varphi(A)}$; il existe un ultrafiltre थ sur $A$, tel que $\lim (\varphi(\mathscr{U}))=b$. Grâce à l'hypothèse de cofinalité, on voit que, pour toute $l \in E^{\prime}$, il existe une partie $U \in \mathcal{Q}$ telle que $l$ soit bornée sur $U$. Donc $\mathscr{U}$ converge vers un élément $c \in A$, tel que $\varphi(c)=b$.

4. Corollaire. Les hypothèses étant les mêmes que celles de la proposition 3, on suppose de plus $\varphi$ surjective. On a alors $(X \in \mathcal{E}) \Leftrightarrow(Y \in \mathcal{L})$.

Preuve. D'après le lemme 1, il suffit de prouver que, pour toute $\mu \in M^{+}(Y)$, il existe $\lambda \in M^{+}(X)$ avec $\mu=\varphi(\lambda)$.

Par l'application $(f \in h(Y)) \leadsto(f \circ \varphi \in h(X))$ on voit que, $\varphi$ étant surjective, $h(Y)$ s'identifie à un sous-espace vectoriel cofinal de $h(X)$, puisque $\left.E^{\prime}\right|_{X}$ est cofinal dans $h(X)$ ([2], 30.13). Il suffit alors d'appliquer le théorème de HahnBanach, pour avoir l'existence de $\lambda$.

Nous allons maintenant nous placer dans le cadre des espaces vectoriels ordonnés.

Nous allons maintenant présenter un schéma différent mais entièrement équivalent à celui du corollaire 4 . 
On se donne un espace vectoriel $V$ (sans topologie), ordonné par un cône convexe saillant $A$, fermé pour la topologie faible la plus fine de $V$, c'est à dire $\sigma\left(V, V^{*}\right)$, et tel que $V=A-A$.

Soit $W$ un sous-espace vectoriel de $V$, tel que, en posant $B=W \cap A$, on ait $W=B-B$ et $B$ cofinal à $A$.

REMARQUE. L'équivalence de ce schéma avec celui du corollaire 4 se constate en prenant $V=E^{\prime}, A=X^{\circ}, W=\varphi^{t}\left(F^{\prime}\right)$.

5. THEOREME. Les cônes $A^{\circ} \subset V^{*}$ et $B^{\circ} \subset W^{*}$ appartiennent à $\delta$, lorsque $V^{*}$ et $W^{*}$ sont munis de $\sigma\left(V^{*}, V\right)$ et de $\sigma\left(W^{*}, W\right)$. On $a\left(A^{\circ} \in \mathfrak{L}\right) \Leftrightarrow\left(B^{\circ} \in \mathfrak{L}\right)$.

Preuve. Les cônes $A^{\circ}$ et $B^{\circ}$ sont évidemment complets; le fait qu'ils sont saillants résulte des relations $V=A-A$ et $W=B-B$.

Soit $\varphi$ l'application canonique de $A^{\circ}$ dans $B^{\circ}$, telle que pour tout $u \in A^{\circ}$, l'élément $v=\varphi(u)$ vérifie $v(b)=u(b)$ pour tout $b \in B$.

Comme $B$ est cofinal à $A$, d'après le théorème de Hahn-Banach, $\varphi$ est surjective; on peut alors appliquer le corollaire 4 avec $X=A^{\circ}, Y=B^{\circ}$ et $\varphi$.

6. Corollaire. Soit $X \in \mathcal{S}$; on $a(X \in \mathfrak{E}) \Leftrightarrow\left(M^{+}(X) \in \mathfrak{E}\right)$.

Preuve. On applique le théorème 5 , en prenant $A=h(X)$ et $B=\left.E^{\prime}\right|_{X}$; on a alors $A^{\circ}=M^{+}(X)$ et $B^{\circ}=X$.

7. Remarque. L'image de $X$ dans $M^{+}(X)$ par l'application $x \rightsquigarrow \varepsilon_{x}$ n'étant pas convexe, l'implication $\left(M^{+}(X) \in \mathcal{L}\right) \Rightarrow(X \in \mathcal{L})$ n'est pas triviale.

Nous allons maintenant donner une caractérisation $(\S 12)$ des chapeaux (§8) d'un cône $M^{+}(X)$; nous en déduirons une caractérisation topologique des cônes $M^{+}(X)$ qui sont éléments de $\mathcal{L}(\S 13)$.

Nous terminerons par un résultat indépendant: La classe $\mathcal{L}$ est stable par produit dénombrable.

8. Definitions. Soit $X \in \delta$; on dit que $K \subset X$ est un chapeau de $X$ ([2], 30.16, $17,18)$ lorsque $K$ est un convexe compact de $X$, tel que que $X \backslash K$ soit convexe. On dit que $X$ est bien coiffé lorsque $X$ est la réunion de ses chapeaux. On dit que $X$ est presque bien coiffé lorsque tout point de $X$ est limite filtrante croissante de points contenus dans des chapeaux de $X$.

$\mathrm{Si}$, de plus, $X$ est réticulé, on sait que $X$ est presque bien coiffé dès que tout point de $X$ est sup. de points contenus dans des chapeaux de $X([4], 2.19)$. 
9. Remarque. Il est bien connu que, si $X$ est bien coiffé, alors $X \in \mathcal{L}$. Par contre ( $X$ presque bien coiffé) n'entraine pas $(X \in \ell)$ : en effet, $\left(\Re^{+}\right)^{I}$ est presque bien coiffé pour tout $I$; si on avait $\left(\Re^{+}\right)^{I} \in E$ pour tout $I$, alors tout $X \in \mathcal{L}$ serait dans $\mathcal{L}$, puisque tout $X \in S$ se plonge dans un $\left(\Re^{+}\right)^{I}([2], 30.10)$.

Cependant, on va voir au $\S 13$ que l'on a, si $X \in \mathcal{S},\left(M^{+}(X) \in \mathcal{L}\right) \Leftrightarrow\left(M^{+}(X)\right.$ presque bien coiffé).

Nous allons donner une caractérisation des chapeaux de $M^{+}(X)$, pour un cône $X \in \mathfrak{s}$.

10. Notations. Soit $X \in \delta$ et $Y$ un exemplaire de $M^{+}(X)$.

Soit $\varphi$ l'application canonique de $Y$ sur $X$ définie par $\varphi(\mu)=r(\mu)=$ (résultante de $\mu$ ); on note encore $\varphi$ l'application induite par $\varphi$ de $M^{+}(Y)$ dans $M^{+}(X)$. Il importe de ne pas confondre, pour $\pi \in M^{+}(Y)$, les deux éléments $r(\pi) \in Y$ et $\varphi(\pi) \in M^{+}(X)$.

11. Proposition. Soit $\mu \in M^{+}(X)$; il existe $\pi \in M^{+}(Y)$ telle que l'on ait ( par abus de langage) $\varphi(\pi)=r(\pi)=\mu$. On peut prendre pour $\pi$, la mesure maximale sur $Y$ qui représente $\mu \in Y$.

Preuve. Si $\mu=\lim _{\mathscr{Q}} \Sigma \varepsilon_{x}$, on vérifie que $\pi=\lim _{\mathscr{Q}_{\mathrm{L}}} \Sigma \varepsilon_{\varepsilon_{\mathrm{x}}}$ convient.

12. Proposition. Soit $K$ une partie de $M^{+}(X)$; les deux propriétés suivantes sont équivalentes:

a) $K$ est un chapeau de $M^{+}(X)$.

b) Il existe une partie compacte $A$ de $X$ telle que $K=(\mu: \mu$ localisable sur $A$ en une mesure de Radon $\geqslant 0$ de masse $\leqslant 1)$.

Preuve. a) $\Leftrightarrow$ b). On va voir qu'on peut prendre $A=\varphi\left(K \cap\left(\varepsilon_{x} ; x \in X\right)\right)$. Désignons par $K_{A}$ le second membre de l'égalité dans b).

1) Soit $\mu \in K$; elle est représentée dans $Y$ par une mesure maximale $\pi$ sur $Y$ (voir $\S 11$ ); on représente $([2], 30.16,17,18) \pi$ par une mesure de Radon $m \geqslant 0$, de masse $\leqslant 1$, sur le compact $K \cap\left(\varepsilon_{x} ; x \in X\right)$; alors $\mu \in M^{+}(X)$ sera représentée par $\varphi(m)$, qui est une mesure de $\operatorname{Radon} \geqslant 0$, de masse $<1$, sur $A$. Donc $\mu \in K_{A}$ et $K \subset K_{A}$.

2) Soit $\mu \in M^{+}(X)$ représentée par une mesure de Radon $m \geqslant 0$ sur $A$, de masse $\leqslant 1$; on a $m=\lim _{\mathcal{Q}_{2}} \sum \alpha_{i} \varepsilon_{x_{i}}$, avec $\Sigma \alpha_{i} \leqslant 1$ et $x_{i} \in A$. Alors $\hat{m}=\lim _{\text {भI }} \Sigma \alpha_{i} \varepsilon_{\varepsilon_{x_{i}}}$ est une mesure de Radon $\geqslant 0$ sur $K$ de masse $\leqslant 1$, qui représente un élément $\pi \in M^{+}(Y)$ tel que $r(\pi)=\mu \in Y$, comme $r(\pi) \in K$ nécessairement, on a $\mu \in K$, donc $K_{A} \subset K$. 
b) $\Rightarrow$ a). Soit $A_{1}=\{x ; x \neq 0, x \in A, \exists k>1, k x \in A\}$; c'est un $G_{\delta}$ (relatif) de $A$, car on a

$$
A_{1}=A \bigcap_{n}([0,1-1 / n] \cdot A)^{C} ; \text { il est alors clair que }
$$

$K=\left\{\mu ; \mu\right.$ localisable sur $A_{1}$ en une mesure de Radon $\geqslant 0$, de masse $\left.\leqslant 1\right\}$. Comme la localisation en une mesure de Radon sur $A_{1}$ est unique ([4] 2.13), on en déduit que $K$ est un chapeau de $M^{+}(X)$ : si $\mu_{1}, \mu_{2} \notin K$ et $\left(\mu_{1}+\mu_{2}\right) / 2 \in K$ on a $\mu_{1}, \mu_{2}$ localisables sur $A_{1}$ par des mesures $m_{1}, m_{2} \geqslant 0$; on a $\left(m_{1}(1)+m_{2}(1)\right) / 2 \leqslant 1$, donc $m_{1}(1) \leqslant 1$ ou $m_{2}(1) \leqslant 1$ - Contradiction-De plus, il est clair que $K$ est un convexe compact.

13. CoROllaire. Soit $X \in S$; les deux propriétés suivantes sont équivalentes:

a) $X \in$ e.

b) $M^{+}(X)$ est presque bien coiffé.

Nous terminons en étudiant la stabilité de la class $\mathscr{L}$.

14. THEOREME. La classe L est stable par produit dénombrable, et donc aussi par limite projective dénombrable.

Preuve. Elle découle des deux lemmes suivants.

15. LeMme. Soit $X_{1}, X_{2}, X_{n}$, une suite de cônes appartenant à 巳et $X=\Pi_{1}^{\infty} X_{i}$. On désigne par $p_{n}$ la projection canonique de $X$ sur $X_{n}$. Pour toute $\mu \in M^{+}(X)$, il existe $\mu_{0} \leqslant \mu$, avec $\mu_{0} \neq 0$ si $\mu \neq 0$ telle que, pour tout $n, p_{n}\left(\mu_{0}\right)$ soit localisable en une mesure de Radon $\geqslant 0$ sur la base $Y_{n} d$ 'un sous cône convexe de $X_{n}$, avec $Y_{n}$ compact.

Preuve. Soit $h \in h(X)$ telle que $\mu(h)=1$. Pour tout $n$ soit $\mu_{n}=$ $\sup \left(\lambda: 0 \leqslant \lambda \leqslant \mu, p_{n}(\lambda)=0\right.$ ); on voit (voir Lemma 2) qu'il existe $\lambda_{n} \leqslant \mu-\mu_{n}$, telle que $\lambda_{n}(h)+\mu_{n}(h) \geqslant 1-1 / 2^{n+1}$, avec $p_{n}\left(\lambda_{n}\right)$ localisable en une mesure de Radon $\geqslant 0$ sur la base $Y_{n}$ compacte d'un sous-cône convexe de $X_{n}$. On voit que l'on a, en posant $\mu_{0}=\inf \left(\lambda_{n}+\mu_{n}\right)$ l'inégalité: $\mu_{0}(h) \geqslant 1 / 2$ : en effet, soit $\nu_{n}=\lambda_{n}$ $+\mu_{n}$; on a $\nu_{1} \wedge \nu_{2}(h)+\nu_{1} \vee \nu_{2}(h)=\nu_{1}(h)+\nu_{2}(h)$, d'où, comme $\nu_{1} \vee \nu_{2} \leqslant \mu$ : $\nu_{1} \wedge \nu_{2}(h) \geqslant(1-1 / 4)+(1-1 / 8)-1=5 / 8$; de même $\nu_{1} \wedge \nu_{2} \wedge \nu_{3}(h)+\left(\nu_{1}\right.$ $\left.\wedge \nu_{2}\right) \vee \nu_{3}(h)=\nu_{1} \wedge \nu_{2}(h)+\nu_{3}(h)$, d'où $\nu_{1} \wedge \nu_{2} \wedge \nu_{3}(h) \geqslant 5 / 8+1-1 / 16-1$ $=9 / 16$, puisque $\left(\nu_{1} \wedge \nu_{2}\right) \vee \nu_{3} \leqslant \mu$. De proche en proche, on a bien $\mu_{0}(h) \geqslant$ $1 / 2$. 
16. Lemme. Sous les conditions du lemme 15 , alors $\mu_{0}$ est localisable en une mesure de Radon $\geqslant 0$ sur un compact de $X$.

Preuve. On notera $\mu$ au lieu de $\mu_{0}$. On peut supposer que $p_{n}(\mu)$ est localisable sur $Y_{n}$ en une mesure de Radon $\geqslant 0$ de masse $\leqslant 1$ et que $Y_{n}=l_{n}^{-1}(1)$ où $l_{n}$ est une forme linéaire continue sur $X_{n}$. On a alors $\sum_{1}^{\infty} l_{n}(r(\mu)) / 2^{n} \leqslant 1$. Soit $\mu=\Sigma \mu_{i}$ une décomposition finie de $\mu$ avec $\mu_{i} \neq 0$ et $\mu_{i} \geqslant 0$. On peut écrire $\mu=\Sigma\left(\mu_{i} / q\left(\mu_{i}\right)\right)$. $q\left(\mu_{i}\right)$ où $q(\lambda)=\Sigma_{1}^{\infty} l_{n}(r(\lambda)) / 2^{n}$, car on a toujours $0<q\left(\mu_{i}\right)<+\infty$ et $\sum q\left(\mu_{i}\right) \leqslant$ 1: En effet, comme $\mu_{i} \leqslant \mu$ on a $r\left(p_{n}\left(\mu_{i}\right)\right) \in$ (cône de base $\left.Y_{n}\right)$, donc $\left(q\left(\mu_{i}\right)=0\right.$ ) entraine $\left(\mu_{i}=0\right)$. En passant à la limite sur les décompositions $\mu=\Sigma \mu_{i}$, on voit que $\mu$ est localisable en une mesure de Radon $\geqslant 0$ sur le compact $\pi_{1}^{\infty} 2^{n}$ conv $\left(0, Y_{n}\right)$, puisque $r\left(\mu_{i}\right) / q\left(\mu_{i}\right)$ appartient à ce compact et que $\sum q\left(\mu_{i}\right) \leqslant 1$.

\section{Bibliographie}

[1] R. Becker, 'Some consequences of a kind of Hahn-Banach Theorem,' Séminaire Choquet, 1977-78, $n^{\circ} 2$.

[2] G. Choquet, Lectures on analysis, Vol. 1-3 (Mathematics Lecture Note Series, Benjamin, New York, Amsterdam, 1969)

[3] H. Dinges, 'Decomposition in ordered semi-groups,' J. Functional Analysis 5 (1970), p. 436-83

[4] A. Goullet de Rugy, 'La théorie des cônes biréticulés,' Ann. Inst. Fourier (Grenoble) 21 (1971).

[5] E. Thomas, 'Integrals representations in conucluar spaces', Vector space measures and applications II. Proceedings, Dublin 1977, edited by R. M. Aaron and S. Dineen, pp. 172-179 (Lecture Notes in Mathematics 645, Springer-Verlag, Berlin, Heidelberg. New York).

\section{Université Paris VI}

Equipe d'Analyse, E.R.A 294

4 Place Jussieu

75230, Paris

France 